\title{
Saturation spectroscopy in optically thick three-level gas media
}

\author{
C. Schmidt-Iglesias, L. Roso, and R. Corbalan \\ Departament de Fisica, Universitat Autonoma de Barcelona, 08193 Bellaterra, Barcelona, Spain
}

Received August 21, 1989; accepted October 23, 1989

\begin{abstract}
High-contrast transmission spectra arising from saturated absorption in optically thick Doppler-broadened threelevel media are analyzed within the formalism of Maxwell-Bloch-type equations. It is found that although in the strong pumping engine propagation-induced line narrowing and light-shift elimination occur, spectral features lying within the natural linewidth cannot be resolved.
\end{abstract}

Saturated absorption spectroscopy (SAS) is a widely used method for obtaining Doppler-free resonances on the absorption of a probe beam when atomic and molecular vapors are simultaneously interacting with a counterpropagating pump beam of the same frequen$\mathrm{cy}^{1}$ (see Fig. 1). The technique is mostly used with modest pump-beam intensities and small number densities in the sample in order to avoid power- and collisional-broadening effects, respectively.

An interesting development of SAS has been achieved recently by Svanberg et al. ${ }^{2,3}$ They have experimentally shown that by working in the regime of strong pumping intensities and optically thick atomic samples, natural linewidth resonances on an essentially zero background can be directly obtained. Theoretical descriptions of the SAS experiments in the new regime have also been given, based either on a simple hole-burning model ${ }^{3}$ or on a more accurate analysis in terms of Maxwell-Bloch-type equations. ${ }^{4}$ Both these models predict that subnatural linewidths can be reached, but since they consider samples of Dopplerbroadened two-level atoms, there still remains the interesting question of whether SAS in the new regime can be used to resolve spectral features that lie within the natural linewidth.

The simplest model that can give us physical insight into the subnatural spectroscopy problem is a threelevel system with two nearly degenerate levels (see Fig. 2). A practical example could be an atom or molecule with a $J=1$ ground state and an excited $J=0$ state placed on a static magnetic field parallel to the direction of the two counterpropagating pump and detection beams, with the $J=1-J=0$ transition frequency $\omega_{0}$ in the absence of external fields being far apart from all other absorption frequencies of the system.

The absorbing gaseous medium composed of such three-level atoms is subjected to irradiation by a monochromatic field, $\mathbf{E}(z, t)$, composed of two counterpropagating plane waves of arbitrary strength,

$$
\begin{gathered}
\mathbf{E}(z, t)=\alpha(z) \exp (-i \omega t)+\text { c.c., } \\
\alpha(z)=\alpha^{+}(z) \exp \left(i k_{0} z\right)+\alpha^{-}(z) \exp \left(-i k_{0} z\right),
\end{gathered}
$$

where $k_{0}=\omega / c$. Note that the amplitudes of the $\alpha^{+,-}$ waves depend on the penetration $\operatorname{depth} z$. Specifically, we consider the simplest setup for $\mathrm{SAS}^{1,2}$ shown in
Fig. 1. The $\alpha^{-}$wave originates by retroreflection of the pump beam $\alpha^{+}$on a back mirror after traversing the atomic sample of length $L$. Each $\alpha$ wave couples to both $0-1$ and $0-2$ transitions with respective detunings $\Delta_{1}=\Delta-\delta$ and $\Delta_{2}=\Delta+\delta$, where $\Delta=\omega-\omega_{0}$. The coupling of the $\alpha^{\mu}$ fields is characterized by the Rabi frequencies $2 \Omega^{\mu}$, which for simplicity are assumed to be equal for both $0-1$ and $0-2$ dipole-allowed transitions.

The medium response to the optical field, taking into account population and coherence effects, is governed by the ensemble-averaged density matrix $\rho(v, z$, $t$ ) whose Fourier components can be expressed in terms of a continued fraction ${ }^{5}$ and have been evaluated within a single-relaxation-rate (single- $\gamma$ ) model.

The induced nonlinear polarization $\mathbf{P}(z, t)$ can be expressed in terms of the steady-state susceptibilities as follows:

$$
\mathbf{P}(z, t)=\epsilon_{0}\left(\chi^{+} \alpha^{+}+\chi^{-} \alpha^{-}\right) \exp (-i \omega t)+\text { c.c., }
$$

where $\epsilon_{0}$ is the vacuum permittivity and the saturated medium susceptibilities for the two counterpropagating waves, $\chi^{+}$and $\chi^{-}$, depend on the two phasematched Fourier components of the velocity-averaged atomic coherence,

$$
\left\langle\rho_{0 j}{ }^{\mu}\right\rangle_{v}=f \frac{\sqrt{\pi} \Omega^{\mu}}{k_{0} u \gamma} P_{0 j}{ }^{\mu},
$$

through

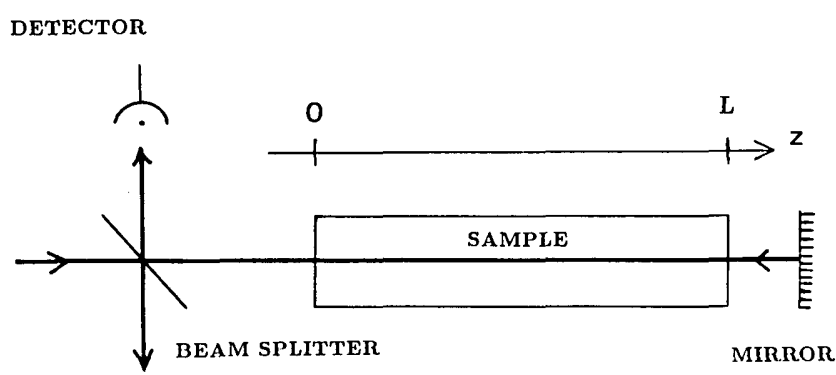

Fig. 1. Simple experimental scheme for SAS in which the probe beam is obtained by retroreflection of the transmitted pump beam, which is strongly attenuated despite considerable bleaching in the optically dense sample. 


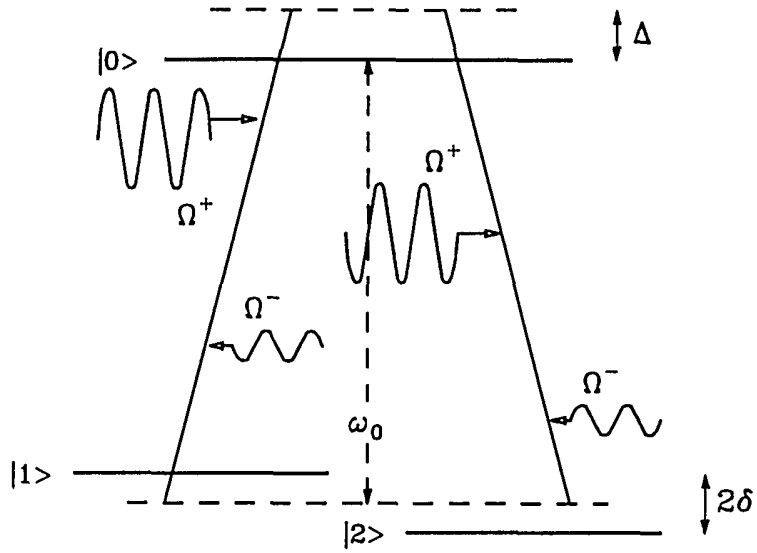

Fig. 2. Three-level system with two nearly degenerate transition frequencies at $\omega_{0}+\delta$ and $\omega_{0}-\delta$, coupled to two counterpropagating waves, with the absorber-field interaction characterized by the half-Rabi frequencies $\Omega^{+}$and $\Omega^{-}$on both transitions.

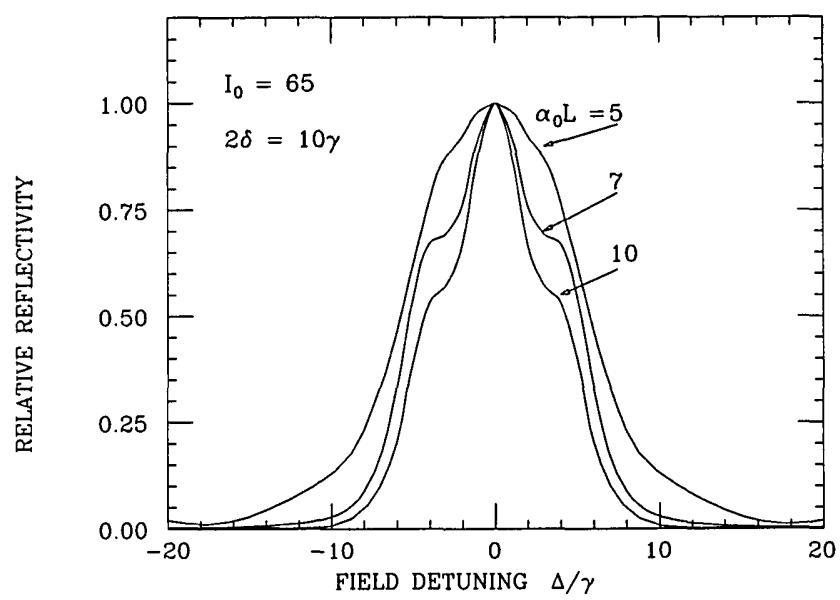

Fig. 3. Spectral line shapes of the probe-beam reflectivity $R$ for different absorption lengths $\alpha_{0} L$, with the sublevel splitting $2 \delta=10 \gamma$. All curves are normalized to unity at the line center.

$$
\chi^{\mu}=\frac{2 \alpha_{0}}{k_{0}}\left(P_{01}{ }^{\mu}+P_{02}{ }^{\mu}\right), \quad \mu=+,-
$$

Here $\alpha_{0}$ is the resonant unsaturated field absorption coefficient given by

$$
\alpha_{0}=\frac{\sqrt{\pi}}{k_{0} u} N f \frac{|d|^{2} \omega}{2 \epsilon_{0} \hbar c \gamma},
$$

where $N$ is the number density of three-level atoms with an equal electric-dipole moment $d$ for both onephoton transitions, equal fractional equilibrium populations $(f=1 / 2)$ in both lower levels, and a Maxwellian velocity distribution with a most probable velocity $u$.

In the slowly varying envelope approximation the spatial evolution of the electromagnetic field is found to satisfy the coupled set of two equations:

$$
\frac{\mathrm{d} \alpha^{\mu}}{\mathrm{d} z}=i \mu \frac{k_{0}}{2} \chi^{\mu} \alpha^{\mu}, \quad \mu=+,-
$$

where $\chi^{\mu}\left(I^{+}, I^{-}\right)$is a function of the penetrationdepth-dependent intensity parameter,

$$
I^{\mu}(z)=\frac{\left|\alpha^{\mu}(z)\right|^{2}}{I_{s}}=4 \frac{\left|\Omega^{\mu}(z)\right|^{2}}{\gamma^{2}},
$$

normalized to the line-center saturation intensity $I_{s}$.

These coupled equations, with boundary conditions $I^{+}(z=0)=I_{0}$, the known incident pump intensity, and $I^{+}(z=L)=I^{-}(z=L)$ for a lossless back mirror, form the basis for the numerical determination of the retroreflected wave, where the quantity of interest is the reflectivity,

$$
\left.R=\left[\alpha^{-}(z=0) / \alpha^{+}=0\right)\right]^{2} .
$$

Figures 3-5 show typical transmission spectra of the retroreflected beam, calculated numerically for different values of the normalized incident pump intensity $I_{0}$, the splitting parameter $2 \delta / \gamma$ of the lower 1 and 2 levels, and the integrated linear absorption coefficient $\alpha_{0} / L$. In addition to the one-photon saturation reso-

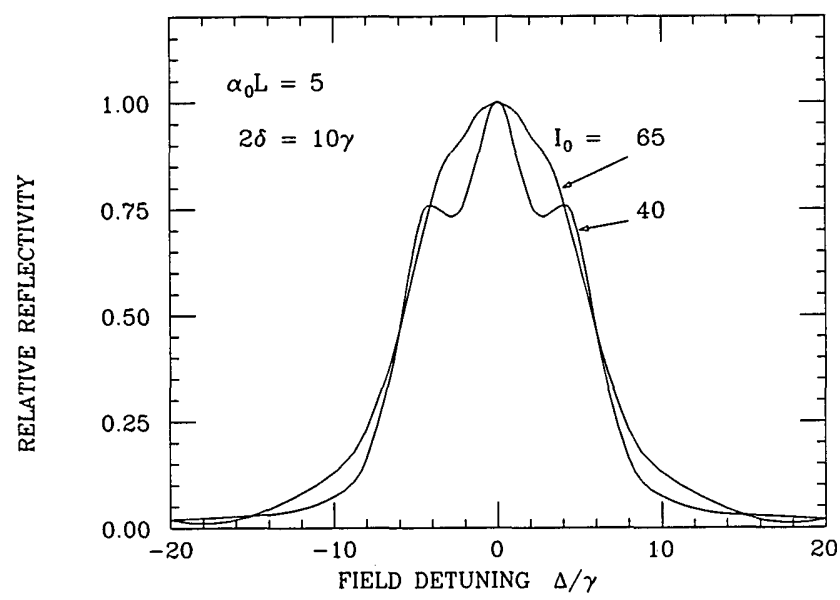

Fig. 4. Normalized probe-beam reflectivity spectra for different incident pump saturation parameters, with $2 \delta=10 \gamma$ and $\alpha_{0} L=5$.

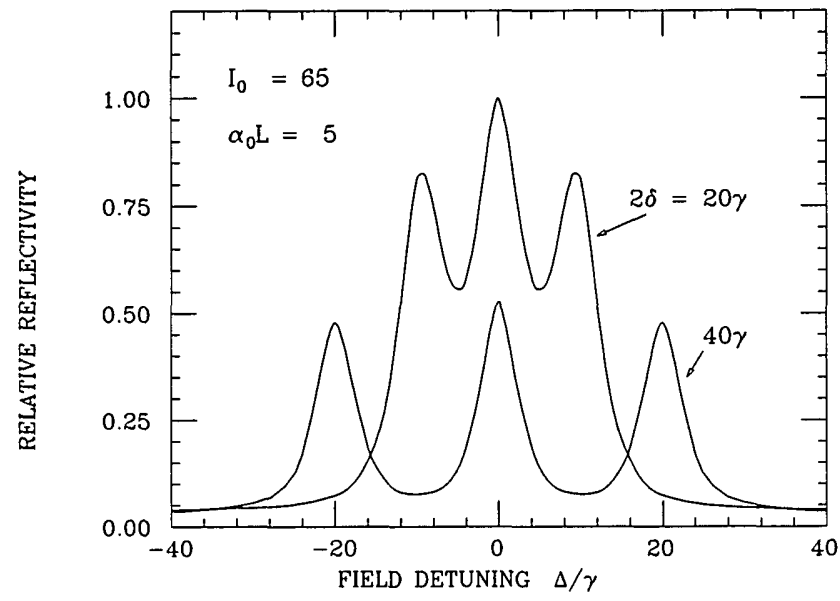

Fig. 5. Probe-beam reflectivity spectra for different sublevel splittings, with the incident pump intensity $I_{0}=65$ and the optical sample thickness $\alpha_{0} L=5$. The applied magnification factor has been chosen to normalize the $2 \delta=20 \gamma$ curve to unity at $\Delta=0$. 
nances, the spectra exhibit at $\Delta=0$ a strong crossover signal where the probe feels through the common excited level the strong bleaching induced by the pump beam.

The details of the line-shape alteration as the optical density increases are shown in Fig. 3 for the case of a highly saturating incident pump beam, $I_{0}=65$. The line-narrowing effect, related to the preferential wing absorption for increasing $\alpha_{0} L$ values, can be clearly seen. However, although power-broadening effects are substantially reduced in dense media, spectral details cannot be resolved even at reduced incident pump levels (see Fig. 4). The saturated absorption resonances only show up as small bumps, even with a splitting between the lower levels as large as 10 times the natural linewidth $(2 \delta / \gamma=10)$. Note that although the resonances overlap, Figs. 3 and 4 already suggest that propagation effects in optically thick media tend to eliminate the incidence of light shifts, ${ }^{6}$ despite having taken into account in our numerical evaluation all the nonlinear effects of coherence. This absence of light shifts appears clearly in the high-contrast and nonoverlapping spectrum of Fig. 5 , in which for $2 \delta=$ $40 \gamma$ the saturation resonances appear exactly centered at $\Delta / \gamma= \pm \delta$.

It thus appears that SAS in the new regime should not be expected to reach the goal of resolving spectral features that lie within the natural linewidth. The new technique gives results that are suited to increase the determination accuracy of the line-center frequency of isolated transitions, with the obvious advantage of annulled light-shift effects.

Interesting discussions with S. Svanberg and L. Sturesson are gratefully acknowledged. This research is supported by the Dirección General de Investigación Científica y Tecnologica (Spain), Project PB0650-C03-01.

\section{References}

1. T. W. Hänsch, I. S. Shanin, and A. L. Schawlow, Phys. Rev. Lett. 27, 707 (1971).

2. S. Svanberg, G.-Y. Yan, T. P. Duffey, and A. L. Schawlow, Opt. Lett. 11, 138 (1986).

3. S. Svanberg, G.-Y. Yan, T. P. Duffey, W.-M. Du, T. W. Hänsch, and A. L. Schawlow, J. Opt. Soc. Am. B 4, 462 (1987).

4. R.Corbalan and L. Roso, in Europhysics Conference Abstracts, J. Heijn and L. Windholz, eds. (European Physical Society, Geneva, Switzerland, 1988), Vol. 12F, p. 138.

5. R. Vilaseca, G. Orriols, L. Roso, R. Corbalan, and E. Arimondo, Appl. Phys. B 34, 73 (1984).

6. M. Himbert, S. Reynaud, J. Dupont-Roc, and C. CohenTannoudji, Opt. Commun. 30, 184 (1979). 